\title{
THE CHANGES IN POWER REQUIREMENTS AND MUSCLE EFFICIENCY DURING ELEVATED FORCE PRODUCTION IN THE FRUIT FLY DROSOPHILA MELANOGASTER
}

\author{
FRITZ-OLAF LEHMANN AND MICHAEL H. DICKINSON* \\ University of California at Berkeley, Valley Life Sciences Building, Department of Integrative Biology, Berkeley, CA \\ 94720, USA
}

Accepted 24 January 1997

\begin{abstract}
Summary
The limits of flight performance have been estimated in tethered Drosophila melanogaster by modulating power requirements in a 'virtual reality' flight arena. At peak capacity, the flight muscles can sustain a mechanical power output of nearly $80 \mathrm{~W} \mathrm{~kg}^{-1}$ muscle mass at $24^{\circ} \mathrm{C}$, which is sufficient to generate forces of approximately $150 \%$ of the animal's weight. The increase in flight force above that required to support body weight is accompanied by a rise in wing velocity, brought about by an increase in stroke amplitude and a decrease in stroke frequency. Inertial costs, although greater than either profile or induced

profile power was approximately twice the induced power at all levels of force generation. Thus, it is the cost of overcoming drag, and not the production of lift, that is the primary requirement for flight in Drosophila melanogaster. By comparing the estimated mechanical power output with respirometrically measured total power input, we determined that muscle efficiency rises with increasing force production to a maximum of $10 \%$. This change in efficiency may reflect either increased crossbridge activation or a favorable strain regime during the production of peak forces.
\end{abstract} power, would be minimal with even modest amounts of elastic storage, and total mechanical power energy should be equivalent to aerodynamic power alone. Because of the large profile drag expected at low Reynolds numbers, the

Key words: fruit fly, Drosophila melanogaster, power requirement, flight, muscle efficiency, aerodynamics, respirometry.

\section{Introduction}

The limits of flight performance are determined by a variety of factors including the maximum mechanical power sustainable by the musculature, the structural constraints of the skeleton and limitations on the production of aerodynamic forces. Because the power requirements for flight are so high compared with other types of locomotory behavior, it has often been assumed that the maximum mechanical power output of the flight muscles places the primary limit on the production of aerodynamic forces (Marden, 1994). The total energetic cost during peak performance is then determined by the efficiency with which the muscles generate mechanical power (Casey, 1981).

Although both the maximum mechanical power output and the efficiency of the locomotory musculature can be estimated from in vitro biophysical experiments, in many instances the values determined from such measurements are clearly lower than the maxima that must occur in flying animals (Ellington, 1991; Josephson, 1993). However, it is difficult to determine maximum mechanical power in vivo because it is necessary to elicit peak locomotory performance under conditions in which one can capture detailed wing kinematics while simultaneously measuring metabolic rate. Several researchers have overcome these difficulties to provide measurements and estimates of peak mechanical output of flight musculature. Marden (1987) used the addition of artificial loads to measure peak flight force in a variety of organisms, and estimated mechanical power from the expected induced power costs. Recently, Dudley (1995) and Chai and Dudley (1995) have measured the peak performance of both insects and hummingbirds by flying them in variable mixtures of helium and oxygen. These studies used a strategy in which an external variable (weight or air composition) was systematically varied until the animals were incapable of sustained hovering flight. Estimates of peak performance can also be made by measuring the kinematics or metabolic rate of animals over a large range of flight speeds, although the estimates from such studies can only be taken as lower limits of performance (Ellington et al. 1990; Wakeling and Ellington, 1997). All these methods, though elegantly noninvasive, are limited in that detailed kinematic and aerodynamic data are difficult to monitor during free flight.

\footnotetext{
*Author for correspondence (e-mail: flymanmd@violet.berkeley.edu).
} 


\section{F.-O. LeHMANn AND M. H. DicKinson}

In this study, a different approach is used, in which we elicit peak performance of tethered Drosophila melanogaster within a 'virtual-reality' flight arena. Although energetic data gathered from tethered animals must be viewed with caution, the analysis allows a precise temporal comparison of the salient experimental parameters. By controlling the vertical motion of the visual world of the fly, we can systematically elicit a regular variation in the generation of total flight force under conditions in which the force, metabolic rate and the kinematic variables required to calculate mechanical power are simultaneously measured. These experiments allow us to estimate the mechanical power capacity of $D$. melanogaster flight muscles and to determine how the various components of maximum mechanical power, as well as muscle efficiency, change with the production of aerodynamic forces.

\section{Materials and methods} Animals

The data within the paper were collected from 27 1- to 4day-old female Canton S wild-type Drosophila melanogaster L. The animals were selected from a laboratory colony maintained at room temperature $\left(22^{\circ} \mathrm{C}\right)$ on Carolina Biological Drosophila medium. We anesthetized the flies by cooling them to approximately $4^{\circ} \mathrm{C}$ on a Peltier stage. The flies were then tethered between the head and the notum to a $5 \mathrm{~mm}$ long, $0.1 \mathrm{~mm}$ diameter tungsten rod using an ultraviolet-activated cement (Crystal Clear, Loctite). The flies were allowed to recover for at least $1 \mathrm{~h}$ before we placed them into the flight arena. At the end of each experiment, we anesthetized each fly with $\mathrm{CO}_{2}$, quickly weighed it, and then killed it (before it recovered from anesthesia) by placing it into a vial of $70 \%$ ethanol. The wings of the flies were later removed and mounted on a microscope slide. We captured an image of the wing, using a compound microscope equipped with a phototube, CCD camera and frame grabber board (Scion LG-3), in a Macintosh Quadra 800 computer. We used NIH image software to measure the length and area of the wings for use in the kinematic estimates of mechanical power. We measured the length and area of several wings before and after storage in alcohol and found no significant shrinkage.

\section{Flight arena}

The flight arena used in this study (Fig. 1) is a more elaborate version of a device that has been previously described (Dickinson and Lighton, 1995). The tungsten rod tethered to the notum of the fly fitted snuggly within the arm of a force transducer that optically tracked the angular deflection of a laser beam aimed at a small mirror on the sensor. The sensor was composed of a $10 \mathrm{~mm}$ long, $0.12 \mathrm{~mm}$ diameter steel wire drawn and fixed taught across two brass supports. A $6 \mathrm{~mm}$ long, $0.32 \mathrm{~mm}$ diameter steel tube was soldered at one end to the center of the wire and acted as the working arm of the sensor. The steel tube was lined with a hollow polyethylene tube that served to hold the $0.1 \mathrm{~mm}$ diameter fly tethers tightly. A $3 \mathrm{~mm} \times 3 \mathrm{~mm}$ front surface mirror was fixed with cyanoacrylate adhesive to the sensor at the intersection of the wire and sensor arm. We aimed a $1 \mathrm{~mW}$ helium-neon laser at the mirror and tracked the motion of the reflected beam at a distance of $50 \mathrm{~cm}$ from the mirror using a SL5-2 spot sensor (UDT Sensors, Inc.). The voltage output of the detector circuitry was linearly proportional to a force applied to the sensor. The sensor was calibrated by applying static loads to it by deflection of an $80 \mathrm{~mm}$ long, $0.1 \mathrm{~mm}$ diameter tungsten beam.

In D. melanogaster, the elevation of the total mean flight force vector is oriented at $24^{\circ}$ with respect to the horizontal body axis, regardless of the animal's orientation in space (Götz and

Fig. 1. Experimental apparatus for simultaneously measuring metabolic output, flight force and wingbeat kinematics in Drosophila melanogaster. (A) Flies are tethered to a flight force transducer that optically tracks the angular deflection of a laser beam aimed at a small mirror mounted to the fly's tether. The fly is enclosed within a flow-through chamber for respirometric measurement. Wing stroke frequency and amplitude are continuously measured by optically tracking the shadows of the two wings cast by an infrared light source. The respirometry chamber was enclosed by a close-packed cylindrical array of independently controlled light-emitting diodes. (B) Horizontal closed-loop stimulus. Under closed-loop conditions, the animals can actively

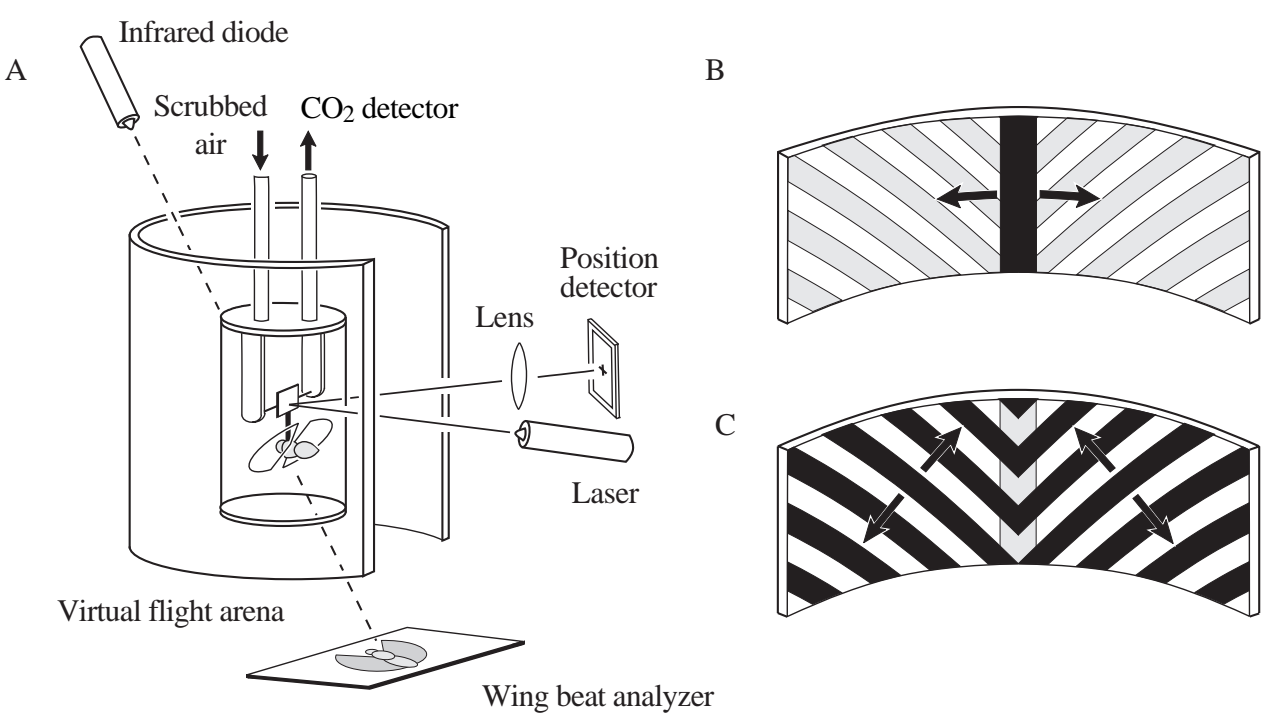
orient towards a $20^{\circ}$ dark stripe pattern by modulating the difference in stroke amplitude between the two wings. (C) Vertical open-loop stimulus. While steering, modulations in total flight force were induced by vertical oscillation of the diagonal stripes. 
Wandel, 1984). This relationship was used to reconstruct the total flight force from the measured component parallel to the body axis. The force sensor and attached fly were enclosed within a $18 \mathrm{ml}$ flow-through respirometry chamber constructed of glass and Plexiglas. The $26 \mathrm{~mm}$ diameter width and $50 \mathrm{~mm}$ depth of the flight chamber was considered sufficiently large to make any performance-enhancing ground effect negligible (Rayner, 1991, 1994). Room air was scrubbed of water and $\mathrm{CO}_{2}$, pulled through the flight chamber at $200 \mathrm{ml} \mathrm{min}^{-1}$ and sampled in a LICOR 6265 gas analyzer. The measurements of $\mathrm{CO}_{2}$ production were transformed into watts assuming a catabolism of sucrose as previously described (Lighton, 1991). Throughout the paper, we present the $\mathrm{CO}_{2}$ data in terms of net metabolic rate, which was calculated by measuring resting metabolic rates before and after each flight sequence and subtracting their average value from the gross values recorded while the animals were flying. During the time at which we measured resting rates, the flies were quiescent and produced little or no motion of their legs.

As the animal flew, its wing stroke frequency and amplitude were continuously measured by optically tracking the shadows of the two wings cast by an infrared light source (Dickinson et al. 1993; Götz, 1987). The respirometry chamber was enclosed by a close-packed cylindrical array of 1536 independently controlled light-emitting diodes (LEDs). By scanning the LED matrix with an appropriate computergenerated pattern, we could create complex visual panoramas that could move both horizontally and vertically around the fly. All experiments were performed under closed-loop conditions in which the fly itself controls the angular velocity of a vertical black bar displayed in the arena by changing the relative stroke amplitudes of its two wings (Fig. 1B). Under these conditions, a fly will actively modulate its wing kinematics in order to stabilize the dark stripe in the frontal position of its visual field (Götz, 1987; Heisenberg and Wolf, 1984). While it actively stabilized the vertical bar, a superimposed pattern of diagonal stripes was oscillated vertically around the fly under open-loop conditions (Fig. 1C). The flies responded robustly to this vertical motion by modulating the production of total flight force. Thus, the experiments are roughly analogous to electronically changing the weight of the animal. During each experiment, we collected seven channels of data: wingbeat frequency, left and right stroke amplitude, flight force, $\mathrm{CO}_{2}$ efflux, the angular position of the vertical stripe and the imposed vertical oscillation of the background. All data channels were sampled continuously at $8.3 \mathrm{~Hz}$ using an AXOTAPE data acquisition system (Axon Instruments). In order to calibrate each flight sequence efficiently, the respirometry chamber was equipped with a magnetically activated 'flight stopper'. This device consisted of a tiny plastic ball at the end of a pin hinged to the side of the chamber. By moving a magnet outside the enclosed chamber, the ball could be made to swing into the wing beat envelope of the fly, causing it to stop flying. Flight could be initiated by blowing air through the inlet of the chamber. In most cases, we recorded two flight sequences from each fly, and the results were combined in the subsequent analysis. The temperature within the respirometry chamber was $23-25^{\circ} \mathrm{C}$.

\section{Power calculations}

The cost of hovering flight consists of three terms: induced, profile and inertial power. These terms were calculated according to the equations of Ellington (1984c) for hovering flight with the modifications described below. All the values and terms used in these calculations are given in Table 1. Unless otherwise stated, all values of power are given in terms of $\mathrm{W} \mathrm{kg}^{-1}$ flight muscle mass. In order to transform body weight-specific power into muscle weight-specific power, we used a flight muscle to body mass ratio of 0.30 . This value was determined by dissecting and weighing the thoraces of 20 freshly killed flies after removing the sternum, nervous system and gut.

\section{Induced power}

Induced power is equal to total flight force multiplied by the mean velocity of the wake. In the case of varying flight force, Ellington's (1984c) equation for induced power, $P_{\text {ind, }}^{*}$ may be modified as follows:

$$
P_{\text {ind }}^{*}=\left(\frac{F_{\mathrm{t}}}{m_{\mathrm{M}} \boldsymbol{g}}\right)\left(\frac{F_{\mathrm{t}}}{2 \rho \Phi R^{2}}\right)^{1 / 2} \kappa,
$$

where $F_{\mathrm{t}}$ is total flight force, $\rho$ is air density, $\Phi$ is stroke amplitude in radians, $R$ is wing length, $\kappa$ is the correction factor required because of the periodic nature of vortex shedding in the wake, $m_{\mathrm{M}}$ is the mass of the flight muscle and $\boldsymbol{g}$ is the gravitational constant. We used a correction factor of 1.28 , calculated from the estimates of Ellington (1984b) and

Table 1. Parameters used in the calculation of induced, profile and inertial power

\begin{tabular}{|c|c|c|}
\hline Symbol & Parameter & Value \\
\hline$m_{\mathrm{b}} \boldsymbol{g}$ & Weight $(\mu \mathrm{N})$ & $10.3 \pm 1.27$ \\
\hline$R$ & Wing length $(\mathrm{mm})$ & $2.47 \pm 0.71$ \\
\hline$S$ & Total wing area $\left(\mathrm{mm}^{2}\right)$ & $3.95 \pm 0.18$ \\
\hline$\kappa$ & Rankine-Froude correction factor & 1.28 \\
\hline$\overline{|\mathrm{d} \hat{\phi} / \mathrm{d} \hat{t}|^{3}}$ & $\begin{array}{l}\text { Mean cube of dimensionless angular } \\
\text { velocity }\end{array}$ & 104.5 \\
\hline$(\mathrm{d} \hat{\phi} / \mathrm{d} \hat{t})_{\max }^{2}$ & $\begin{array}{l}\text { Square of maximum dimensionless } \\
\text { angular velocity }\end{array}$ & 30.3 \\
\hline$\hat{h}$ & Dimensionless wing thickness & $5.4 \times 10^{-4}$ \\
\hline$\hat{v}$ & Dimensionless virtual mass & 1.146 \\
\hline$\hat{r}_{3}^{3}(S)$ & Third moment of wing area & 0.242 \\
\hline$\hat{r}_{2}^{2}(m)$ & Second moment of wing mass & 0.345 \\
\hline$\hat{r}_{2}^{2}(v)$ & Second moment of wing virtual mass & 0.342 \\
\hline$\rho_{\mathrm{w}}$ & Wing density $\left(\mathrm{kg} \mathrm{m}^{-3}\right)$ & 1200 \\
\hline$\rho$ & Air density $\left(\mathrm{kg} \mathrm{m}^{-3}\right)$ & 1.2 \\
\hline
\end{tabular}

The values for the first three terms represent the means \pm S.D. of all 27 flies. For the last 10 terms, a single value was used for all flies (see Materials and methods section for details).

For definitions, see Ellington (1984a). 
visualizations of the wake during tethered flight (Dickinson and Götz, 1996).

\section{Profile power}

The calculation of profile power $\left(P_{\text {pro }}^{*}\right)$ is determined by the product of profile drag and the velocity of the wing in the relative stroke plane, which is defined as the plane parallel to the shed vortex loops and perpendicular to the axis of the wake. Using the estimates of Ellington (1984c), we calculated an angular difference between the stroke plane and relative stroke plane of less than $5^{\circ}$ for both strokes, which is consistent with both a flow visualization (Dickinson and Götz, 1996) and an anemometric map of the wake (Lehmann, 1994). This small difference can be ignored, and we have calculated profile power on the basis of the product of the mean profile drag coefficient, $\overline{C_{\mathrm{D}, \mathrm{pro}}}$, and the velocity of the wing within the stroke plane (after Ellington, 1984c):

$$
P_{\text {pro }}^{*}=\frac{\rho S n^{3} \Phi^{3} R^{3} \hat{r}_{3}^{3}(S) \overline{|\mathrm{d} \hat{\phi} / \mathrm{d} \hat{t}|^{3}}}{16 m_{\mathrm{M}} \boldsymbol{g}} \overline{C_{\mathrm{D}, \text { pro }}},
$$

where $n$ is stroke frequency, $S$ is the surface area of the two wings, $\hat{r}_{3}^{3}(S)$ is the dimensionless third moment of wing area, and $\overline{|\mathrm{d} \hat{\phi} / \mathrm{d} \hat{t}|^{3}}$ is the mean cube of the absolute value of dimensionless angular velocity. The values for wing length and area were determined by measurement of the excised wings after each experiment. We determined $\hat{r}_{3}^{3}(S)$ for a sample of eight wings of varying size and found the standard deviation to be less than $2 \%$ of the mean. In addition, the values were independent of wing length. For these reasons, we did not measure $\hat{r}_{3}^{3}(S)$ for each fly, but rather used the mean value of 0.242 determined from the eight sample wings.

The dimensionless angular velocity was estimated from the published kinematics of tethered flight in D. melanogaster and two simple models of the wing stroke. In the sawtooth model, the wing tip velocity is constant during both half-strokes. In the harmonic model, the wing tip follows a half-cosine curve during both half-strokes. In both models, the downstroke takes up $62 \%$ of the stroke cycle, in accordance with the kinematic studies of Zanker (1990a) and Lehmann (1994). The actual stroke kinematics reported by Zanker and Lehmann lie somewhere within the extremes represented by the two models. Consequently, we used a value of 104.6 for $\overline{|\mathrm{d} \hat{\phi} / \mathrm{d} \hat{t}|^{3}}$ based on the average of the two calculations. The mean profile drag coefficient, $\overline{C_{\mathrm{D} \text {,pro }}}$, was estimated as $7 /(\sqrt{R e})$, where $R e$ is Reynolds number, according to the approximation of Ellington (1984c), with the mean Reynolds number throughout the stroke calculated as:

$$
R e=\frac{S}{v} n \Phi,
$$

where $v$ is kinematic viscosity. Since the last two terms (stroke frequency and amplitude) varied throughout each flight sequence, we calculated $\overline{C_{\mathrm{D} \text {,pro }}}$ at each time point, with values ranging from approximately 0.5 to 0.7 . If anything, these values probably provide a conservative estimate of profile power. The angle of attack used during both the upstroke and downstroke in D. melanogaster is approximately $40^{\circ}$ (Zanker, $1990 a$ ). At this angle, the steady-state drag coefficient of a $D$. virilis wing $(R e=200)$ is 0.8 (Vogel, 1967), and the steady and transient unsteady drag coefficients of a two-dimensional model Drosophila wing $(R e=128)$ are 1.5 and 2, respectively (Dickinson and Götz, 1993).

\section{Inertial power}

The mean inertial power $\left(P_{\mathrm{acc}}^{*}\right)$ needed to accelerate the wings and added mass for each stroke is equal to the kinetic energy of the wings at mid-stroke divided by the quarter-stroke acceleration period. This may be calculated from (after Ellington, 1984c):

$$
P_{\mathrm{acc}}^{*}=\frac{\rho S n^{3} \Phi^{2} R^{3}(\mathrm{~d} \hat{\phi} / \mathrm{d} \hat{t})_{\max }^{2}}{2 m_{\mathrm{M}} \boldsymbol{g}}\left[\frac{\rho_{\mathrm{w}}}{\rho} \hat{h} \hat{r}_{2}^{2}(m)+\frac{\pi S \hat{\mathrm{v}} \hat{r}_{2}^{2}(v)}{8 R^{2}}\right],
$$

where $(\mathrm{d} \hat{\phi} / \mathrm{d} \hat{t})_{\max }^{2}$ is the square of the maximum dimensionless wing velocity. We calculated a value of 30.3 for $(\mathrm{d} \hat{\phi} / \mathrm{d} \hat{t})_{\max }^{2}$ from the average value of the sawtooth and harmonic models of the wing stroke as described above. The two terms in the brackets represent the moments of inertia for the wing mass and virtual mass. These are determined by $\rho_{\mathrm{w}}$, the density of cuticle, $\hat{h}$, the dimensionless wing thickness, $\hat{r}_{2}^{2}(m)$, the dimensionless second moment of wing mass, $\hat{v}$, the dimensionless virtual mass, and $\hat{r}_{2}^{2}(v)$, the dimensionless second moment of virtual mass. Methods for deriving all these terms may be found in Ellington (1984a).

The variable most prone to measurement error in the calculation of inertial power is $\hat{h}$, which is found from the ratio of wing mass to wing area. The fluid from fly wings evaporates rapidly, and the mass of a single Drosophila wing approaches the limit of resolution of most analytical balances. To overcome these difficulties, we employed the following strategy to measure wing mass. We anesthetized 10 males and 10 females from our laboratory stocks using $\mathrm{CO}_{2}$. The wings of all the animals ( 40 in total) were quickly excised and placed within a thin-walled $0.2 \mathrm{ml}$ polymerase chain reaction (PCR) tube (Midwest Scientific). Before transferring the wings, we used a piezoelectric discharge to remove accumulated static electricity from the surface of each vial, and the transfer procedure was performed on a grounded, antistatic surface. To minimize water loss, the lid of the PCR tube was kept open only while transferring each wing. The sealed tube with 40 wings was then weighed on an analytical balance to the nearest $0.01 \mu \mathrm{g}$. We then removed the wings and weighed the empty vial. The 40 wings were then mounted side by side in mineral oil on a microscope slide. Using the video microscopy arrangement described above, we calculated the area of each wing and compiled a total wing area for the sample. The total mass of the sample was then divided by the total area to calculate the mass per unit wing area. We made five such measurements of D. melanogaster wings. As part of a more comprehensive comparative study of drosophilid flies (M. H. Dickinson and F.-O. Lehmann, in preparation), we have made 
similar analyses of six additional congeneric species representing a threefold range of body size. The results indicate that the wing mass per unit area is isometric throughout the genus and that dimensionless wing thickness is, therefore, independent of body size through the sampled range of species. In this study, we use a value for $\hat{h}\left(5.4 \times 10^{-4}\right)$ that represents the mean of all these comparative measurements.

The remaining parameters in equation $4, \hat{r}_{2}^{2}(m)$ and $\hat{r}_{2}^{2}(v)$, were calculated from eight sample wings of varying size. Again, the standard deviation was less than $2 \%$ of the mean, and we used the mean values from this sample population in all calculations.

Aerodynamic power $\left(P_{\text {aero }}^{*}\right)$ is the sum of profile and induced power and represents the total work done by the wings on the air. The total power required of the flight muscles, $P_{\text {mech }}^{*}$, depends on $P_{\text {aero }}^{*}, P_{\text {acc }}^{*}$ and the amount of elastic storage, $\alpha$, within the thorax (Dickinson and Lighton, 1995):

$$
P_{\text {mech }}^{*}=\frac{1}{2}\left[\left(P_{\text {aero }}^{*}+\beta P_{\text {acc }}^{*}\right)+R\left(P_{\text {aero }}^{*}-\beta P_{\text {acc }}^{*}\right)\right],
$$

where $\beta=1-\alpha$, and $R$ is a rectification function such that $R(x<0)=0$. Muscle efficiency, $\eta$, may then be calculated from the ratio of $P_{\text {mech }}^{*}$ and $P_{\mathrm{CO}_{2}}^{*}$, the respirometric measure of total flight cost.

\section{Free flight assay}

To test that our tethered flight studies provided a reasonable estimate of free flight performance, we determined the maximum load that freely flying females could carry following take-off. This technique was developed by Marden (1987) in his comparative study, although modifications were required to use this approach for animals as small as D. melanogaster. To increase the load on each individual, we glued a short section of tungsten wire under the abdomen, just below the expected center of gravity for a body orientation typical of hovering flight (David, 1978). We placed individuals under an inverted 21 beaker and induced them to fly by 'chasing' them around with a bent paperclip. We scored 51 animals on their ability to remain airborne for at least $1 \mathrm{~s}$ after take-off while carrying the added load. We made no attempt to change the load on individual flies. Instead, we used a variety of different weights in order to derive the performance from the response of the population as a whole.

\section{Results}

Throughout all the arena experiments, the flies fixated on a black vertical stripe, the angular velocity of which was controlled by the amplitude difference between the left and right wings. In order to introduce regular modulation of total flight force, we oscillated a superimposed pattern of diagonal stripes in the horizontal direction. A typical response of a fly to these combined horizontal closed-loop and vertical open-loop conditions is shown in Fig. 2. As the background pattern moves up and down, the fly modulates its total flight force in an attempt to stabilize the retinal slip in the vertical direction. As long as the flies were under closed-loop control, the response to the horizontal motion was robust and stable. We found no evidence for attenuation of the response over time, unless the fly ceased flying completely. The mean flight duration was $15 \pm 6 \mathrm{~min}$ (mean \pm S.D., $N=48$ sequences from 27 flies), although most flights were terminated deliberately for the purpose of calibration. Important kinematic and energetic parameters during the minimum, maximum and hovering force production are shown in Table 2.

The modulation in flight force was accompanied by regular changes in both stroke amplitude and stroke frequency. However, while stroke amplitude always tracked flight force quite closely, we often observed a rectification of stroke frequency when the animals generated the highest flight forces. The relationships among these parameters are more clearly seen by plotting instantaneous flight force against stroke amplitude and stroke frequency (Fig. 3). Although flight force increases monotonically with stroke amplitude, there is a distinct kink in the correlation such that total flight force rises more gradually with amplitude at forces above body weight (Fig. 3A). The highest flight forces were produced at stroke amplitudes of $170 \pm 8^{\circ}$, which is probably close to the morphological limit for the ventral excursion of the wings (throughout the rest of the manuscript, all values are expressed as mean \pm S.D., $N=27$ ).
Fig. 2. Representative flight sequence during horizontal oscillation of the lift stimulus. Throughout the entire sequence, the fly actively modulates the left-right difference in stroke amplitude in order to stabilize the horizontal position of the vertical stripe under closed loop control. In an attempt to stabilize the superimposed vertical motion, the fly also varies its production of total flight force. This force modulation is accomplished by changes in both stroke amplitude and stroke frequency. Notice the rectification of stroke frequency when the fly generates the highest flight forces.

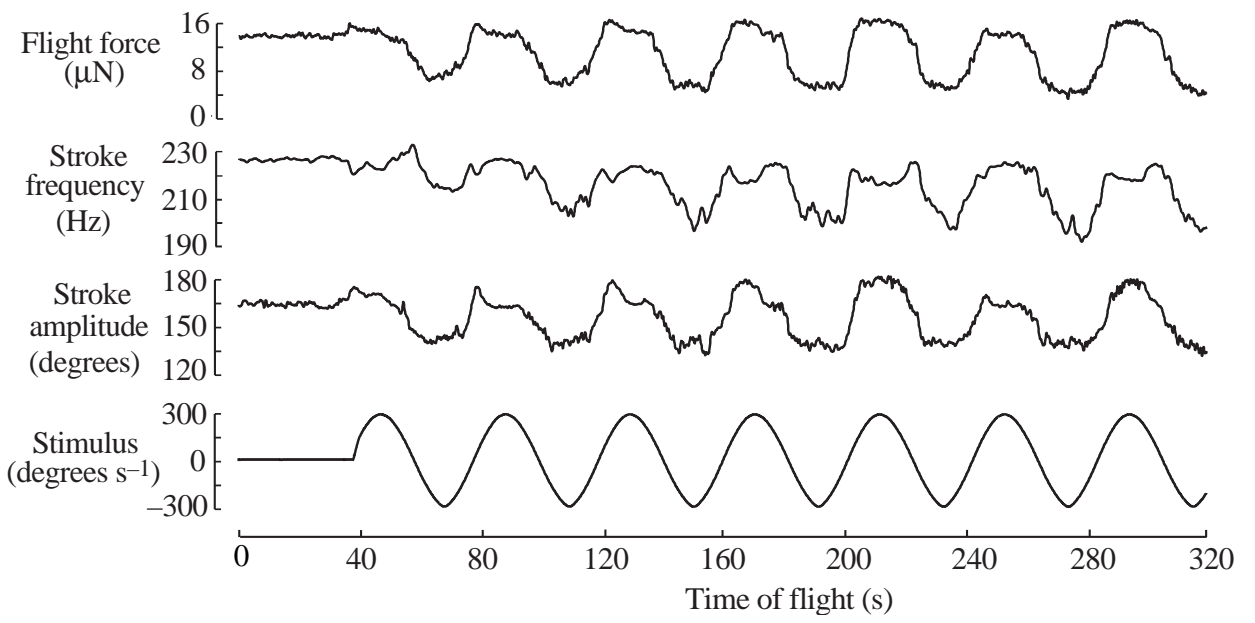




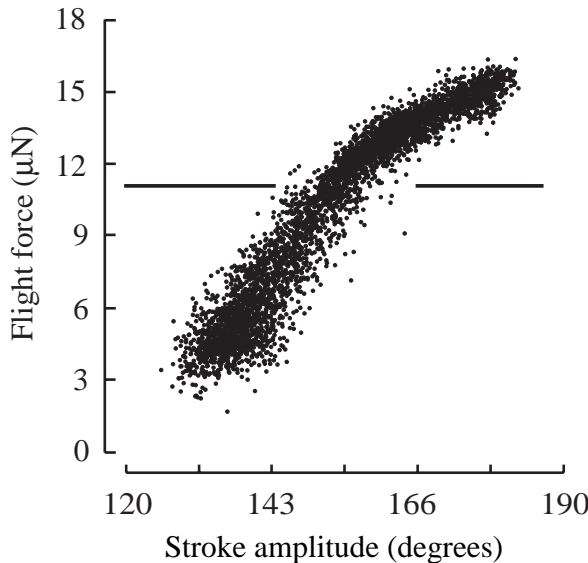

B

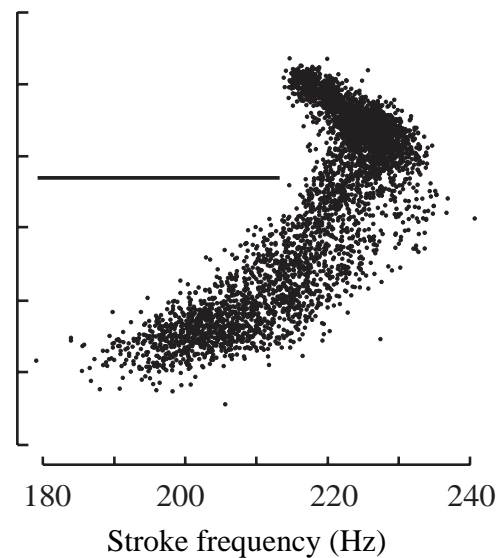

$\mathrm{C}$
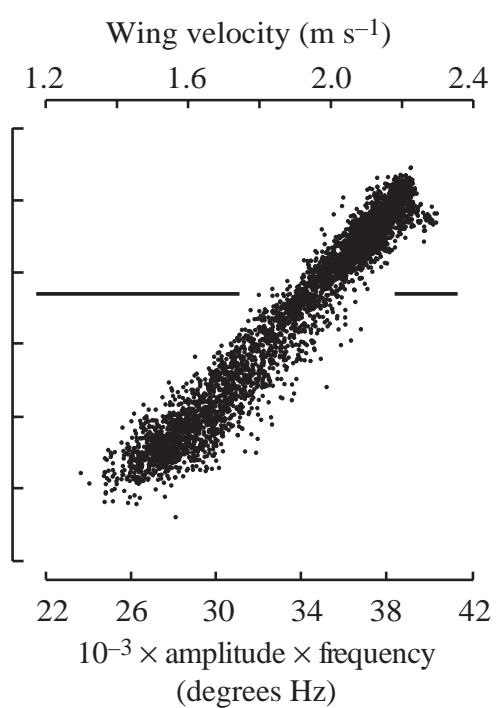

Fig. 3. Data from an individual fly indicating a typical relationship between total flight force and stroke kinematics. (A) Instantaneous flight force plotted against stroke amplitude. Force increases monotonically with amplitude, although the relationship changes slope at high force values. (B) Instantaneous flight force plotted against stroke frequency. Frequency and force are negatively correlated at high force values. (C) The product of stroke amplitude and frequency plotted against force. The top ordinate gives the mean translational velocity of the center of area of the wing. The solid horizontal line in all panels indicates the body weight of the fly.

Thus, as has recently been suggested for hummingbirds (Chai and Dudley, 1995), the maximum flight force in $D$. melanogaster may be constrained in part by the morphological limit of the wing stroke. In contrast to the monotonic increase of flight force with stroke amplitude, the relationship between flight force and stroke frequency is more complex (Fig. 3B). Flight force is positively correlated with stroke frequency at low forces but negatively correlated at high forces. The transition from the positive to negative correlation typically occurred within the range of forces above that required to support body weight. Thus, the generation of peak forces was achieved by an increase in stroke amplitude but a slight decrease in stroke frequency. This kinematic change is quite clear in the time series data in Fig. 2. The puzzling relationship between amplitude and frequency is partially explained by considering the product of the two parameters, which is proportional to the average translational velocity of the wing during flight (Fig. 3C). For all the flies tested, the decreases or saturations in stroke frequency were compensated for by steeper increases in stroke amplitude, such that their product rose linearly with increasing flight force (mean $r^{2}$ value $0.76 \pm 0.19, P<0.001$ ). Flight force was also correlated with the square of wing velocity (mean $r^{2}$ value $0.77 \pm 0.16, P<0.001)$ as is expected in quasi-steady aerodynamic models of flight. However, the intercept of flight force at zero velocity was significantly positive in all cases. This result indicates that, although most of the change in flight force is brought about by an increase in wing velocity, some of the change must be due to an increase in the aerodynamic performance of the wings. The aerodynamic implications of these results will be discussed in a subsequent paper (F.-O. Lehmann and M. H. Dickinson, in preparation).

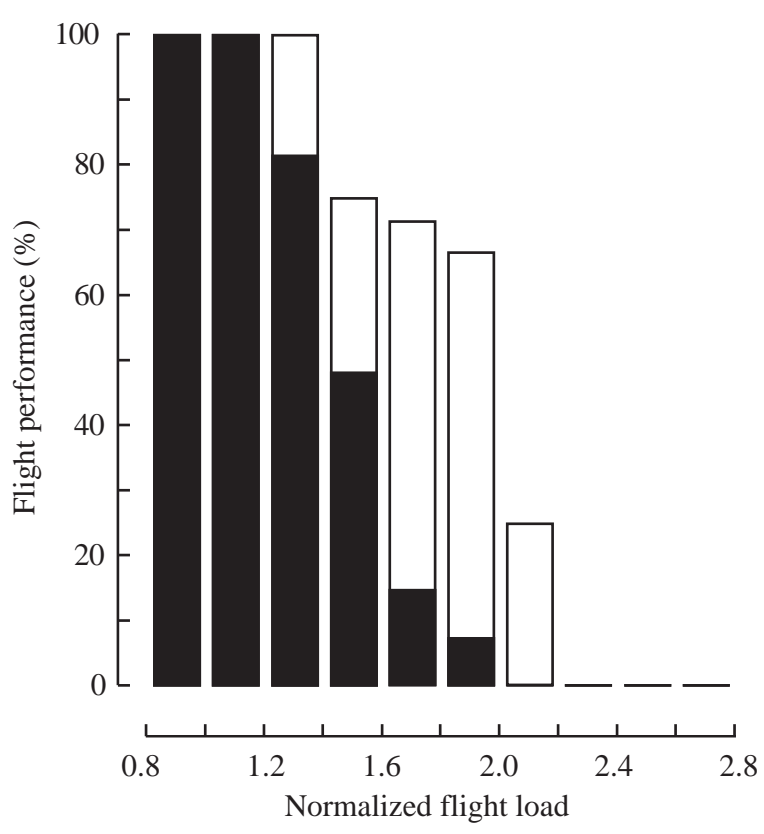

Fig. 4. Comparison of arena performance with that of freely flying animals. Fifty-one flies were scored on their ability to hover for a minimum of $1 \mathrm{~s}$ while carrying a small load glued under their abdomen. The percentages of flies that could sustain flight within each range of load values are indicated by the open bars. The total flight load (the sum of body weight plus the added load) has been normalized to body weight. The experiments were carried out at an ambient temperature of $25^{\circ} \mathrm{C}$. The distribution of peak force values measured within the flight arena $(N=27)$ is indicated by the filled bars. 
Fig. 5. Mass-specific metabolic rate $\left(P_{\mathrm{CO}_{2}}^{*}\right)$ and the three components of mechanical power (induced power $P_{\text {ind }}^{*}$, profile power

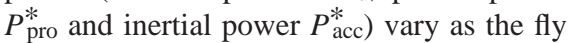
modulates flight force in response to a visual stimulus. All power terms are expressed in terms of flight muscle mass.

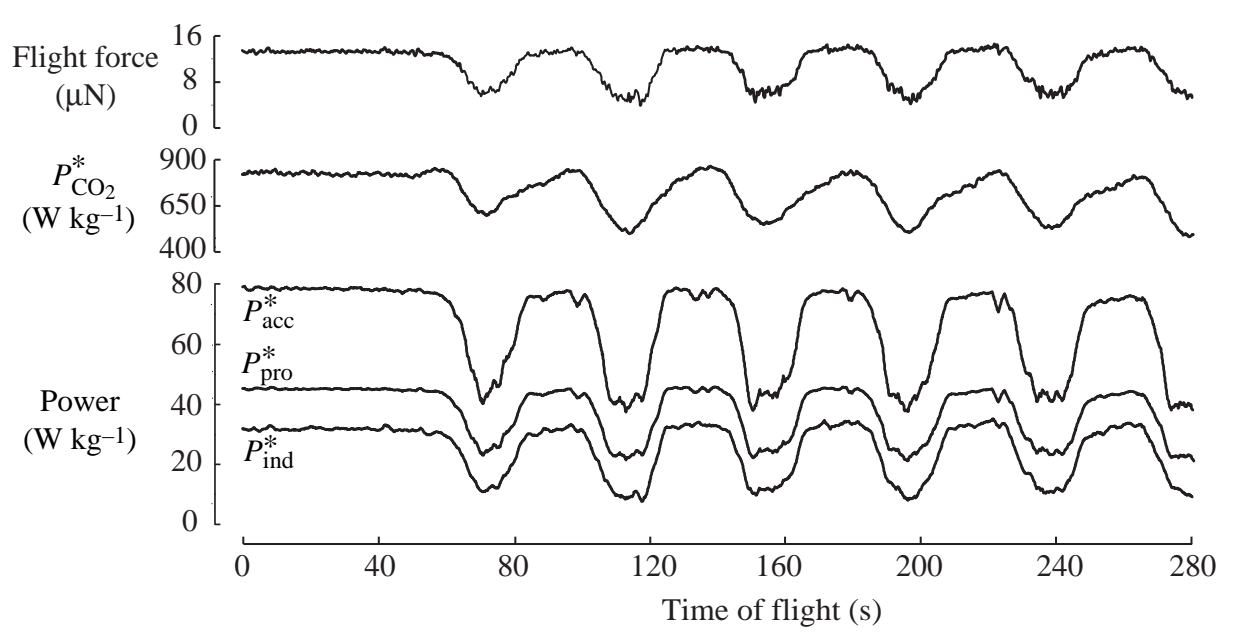

Comparison of arena and free-flight performance

Under the conditions described above, the flies generated peak forces of $134 \pm 20 \%$ of body weight. The highest value generated by a single fly was $185 \%$. Since tethered flight studies have been criticized as not accurately reflecting the flight behavior of freely flying animals (Dudley, 1990; Kammer and Heinrich, 1978), we compared these results with maximum loads in free flight. In free flight, no fly (0/13) could support a total load that exceeded $220 \%$ of its body weight (the weight of the fly plus an additional load of $120 \%$ ). Only $25 \%$ of the flies (2/8) were capable of supporting loads greater than $200 \%$. In general, the freely flying flies could carry loads that were approximately $20 \%$ greater than the maximum forces produced by tethered flies within the arena (Fig. 4). Thus, the most conservative view is that the peak performance in the flight arena was only $80 \%$ of what can be achieved in free flight. However, there are several reasons to expect that the peak muscle performance within the arena may be comparable to that for free flight conditions even if maximum force production appears to be lower. First, in most instances, our procedure elicited wing stroke amplitudes that probably represent the morphological limit for D. melanogaster. Second, the free flight evaluations only required that the flies remain airborne for $1 \mathrm{~s}$ after initiating flight. In contrast, the measurements of maximal flight force in the arena were started at least $15 \mathrm{~s}$ after the onset of flight, in order to allow the respirometry readings to stabilize fully. Third, in the free flight experiments, the flies rarely hovered in place when carrying added loads. This forward velocity of the animals should augment force production by contributing to the translational velocity of the wings. The arena paradigm, in contrast, represents a particularly challenging case of an infinitely small advance ratio in which there is no forward motion of the body and all of the wing velocity comes from flapping and induced flow. However, the requirements in free flight might be higher as a result of the additional cost of parasite drag. We estimated the drag force of D. melanogaster flying at $0.5 \mathrm{~m} \mathrm{~s}^{-1}$ to be $0.5 \mu \mathrm{N}$, based on the drag coefficients of the body measured by Vogel (1966) for $D$. virilis. A flight speed of $0.5 \mathrm{~m} \mathrm{~s}^{-1}$ is at the high end of the speeds observed in free flight for a larger species, $D$. hydei. Therefore, the added cost of body drag in the free flight experiments would probably account for no more than a $5 \%$ increase in the total mechanical power, whereas the aerodynamic advantage of forward velocity is likely to be much greater. For example, the addition of a $0.5 \mathrm{~m} \mathrm{~s}^{-1}$ forward velocity would increase the relative velocity of the wings during the downstroke by approximately $30 \%$. For these reasons, we are confident that the underlying physiological limits of the flight muscles measured within the arena are reasonably close to those that would occur in free flight.

Table 2. Flight parameters measured during extremes of force production

\begin{tabular}{|c|c|c|c|c|c|c|c|c|c|}
\hline & $\begin{array}{c}F_{\mathrm{t}} / m_{\mathrm{b}} \boldsymbol{g} \\
(\%)\end{array}$ & $\begin{array}{c}\Phi \\
\text { (degrees }\end{array}$ & $\begin{array}{c}n \\
(\mathrm{~Hz})\end{array}$ & $\begin{array}{c}P_{\mathrm{CO}_{2}}^{*} \\
\left(\mathrm{~W} \mathrm{~kg}^{-1}\right)\end{array}$ & $\begin{array}{c}P_{\text {ind }}^{*} \\
\left(\mathrm{Wg}^{-1}\right)\end{array}$ & $\begin{array}{c}P_{\text {pro }}^{*} \\
\left(\mathrm{~W} \mathrm{~kg}^{-1}\right)\end{array}$ & $\begin{array}{c}P_{\text {aero }}^{*} \\
\left(\mathrm{~W} \mathrm{~kg}^{-1}\right)\end{array}$ & $\begin{array}{c}P_{\mathrm{acc}}^{*} \\
\left(\mathrm{~W} \mathrm{~kg}^{-1}\right)\end{array}$ & $\begin{array}{c}\eta \\
(\%)\end{array}$ \\
\hline Minimum & $44 \pm 23$ & $148 \pm 9$ & $190 \pm 18$ & $519 \pm 123$ & $7.1 \pm 5.3$ & $24.7 \pm 6.8$ & $31.8 \pm 10.8$ & $43.5 \pm 13.5$ & $6.1 \pm 1.3$ \\
\hline Hovering & 100 & $162 \pm 8$ & $209 \pm 15$ & $664 \pm 100$ & $21.4 \pm 1.0$ & $38.4 \pm 6.3$ & $59.8 \pm 6.4$ & $67.4 \pm 13.5$ & $9.1 \pm 1.2$ \\
\hline Maximum & $134 \pm 20$ & $169 \pm 7$ & $212 \pm 12$ & $727 \pm 119$ & $32.4 \pm 6.1$ & $44.6 \pm 8.6$ & $77.0 \pm 12.4$ & $77.0 \pm 16.5$ & $10.7 \pm 1.2$ \\
\hline
\end{tabular}

For each fly, we calculated the mean values of all the data points within the flight recording that fell within the top $1 \%$ (maximum) or bottom $1 \%$ (minimum) of flight force or within $1 \%$ of body weight (hovering).

$\Phi$, stroke amplitude; $n$, stroke frequency; $F_{\mathrm{t}} / m_{\mathrm{b}} \mathrm{g}$, flight force per body weight; $P_{\text {ind }}^{*}$, induced power; $P_{\text {pro }}^{*}$, profile power; $P_{\text {acc }}^{*}$, inertial power; $P_{\text {aero }}^{*}$, aerodynamic power; $P_{\mathrm{CO}_{2}}^{*}$, total flight cost measured using respirometry; $\eta$, muscle efficiency.

All values represent the mean \pm S.D. $(N=27)$. 

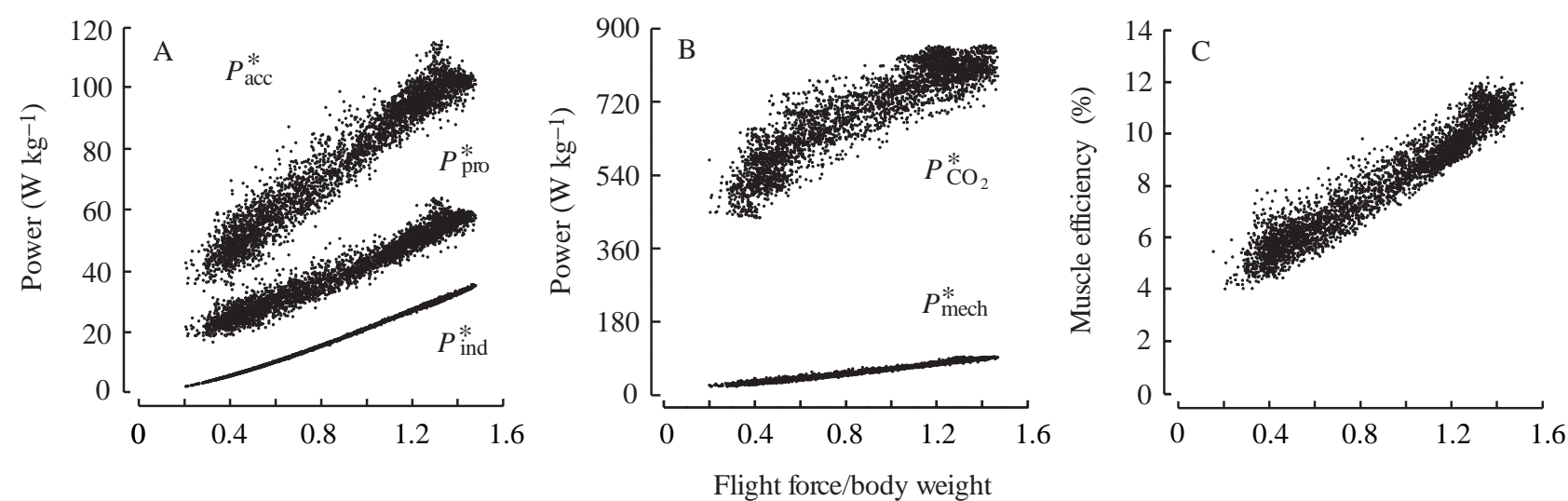

Fig. 6. Data from an individual fly indicating the typical changes in $P_{\mathrm{CO}_{2}}^{*}, P_{\text {mech }}^{*}$ and muscle efficiency with increasing flight force. (A) All three components of mechanical power increase with increasing flight force. (B) $P_{\text {mech }}^{*}$ and $P_{\mathrm{CO}_{2}}^{*}$ plotted against flight force. $P_{\text {mech }}^{*}$ was calculated assuming an elastic storage of at least $15 \%$. (C) Muscle efficiency rises with increasing flight force.

\section{Power requirements}

The mechanical power requirements for hovering flight consist of three components: induced, profile and inertial power, all of which could potentially contribute to an elevation in total metabolic cost with the increasing flight force (Casey, 1981). During flight, the magnitude of these power requirements fluctuates as the animals modulate the production of total flight force in response to the oscillatory stimulus (Fig. 5; Table 2). As shown in Fig. 6A, values for all three power terms increase monotonically with increasing flight force. The magnitude of $P_{\text {acc }}^{*}$ is greatest, followed by $P_{\text {pro }}^{*}$ and

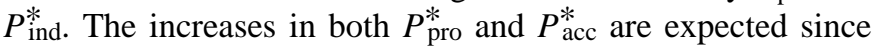
both terms grow as power functions of wing velocity, which rises with increasing flight force (Fig. $3 \mathrm{C}$ ). The rise in $P_{\text {pro }}^{*}$ is not as steep as one might expect from its cubic dependence on wing velocity. This is because the increase in velocity causes an increase in the Reynolds number and a corresponding drop in drag. Over the full range of flight forces, the estimated value of $\overline{C_{\mathrm{D}} \text {,pro }}$ decreased by roughly $20 \%$, attenuating the rise in $P_{\text {pro. Induced power also grows with increasing flight force as }}^{*}$ expected from equation 1. However, it should be noted that, since flight force was strongly correlated with stroke amplitude (and therefore the area swept by the wings), the Rankine-Froude wake velocity term does not contribute substantially to the total increase in $P_{\text {ind }}^{*}$.

The total mechanical power requirement for flight, $P_{\text {mech, }}^{*}$ is not simply the sum of $P_{\text {aero }}^{*}$ and $P_{\text {acc }}^{*}$, but depends on the amount of elastic storage within the muscles and skeleton of
Fig. 7. Flight kinematics appear to be constrained by power availability and the morphological limit of stroke amplitude. The instantaneous value of stroke frequency has been plotted as a function of instantaneous stroke amplitude during a flight sequence from one individual. Instantaneous flight force (normalized to body weight) has been encoded in pseudocolor as indicated by the scale on the right. The hyperbolic lines superimposed over the data points represent mechanical power isolines. The distribution of possible frequency and amplitude combinations is limited by the maximum power output of the flight musculature. The highest flight forces are produced towards the right-hand side of the distribution and may be constrained by the morphological limits of stroke amplitude. The data shown come from a single fly but are representative of nearly all the individuals tested.

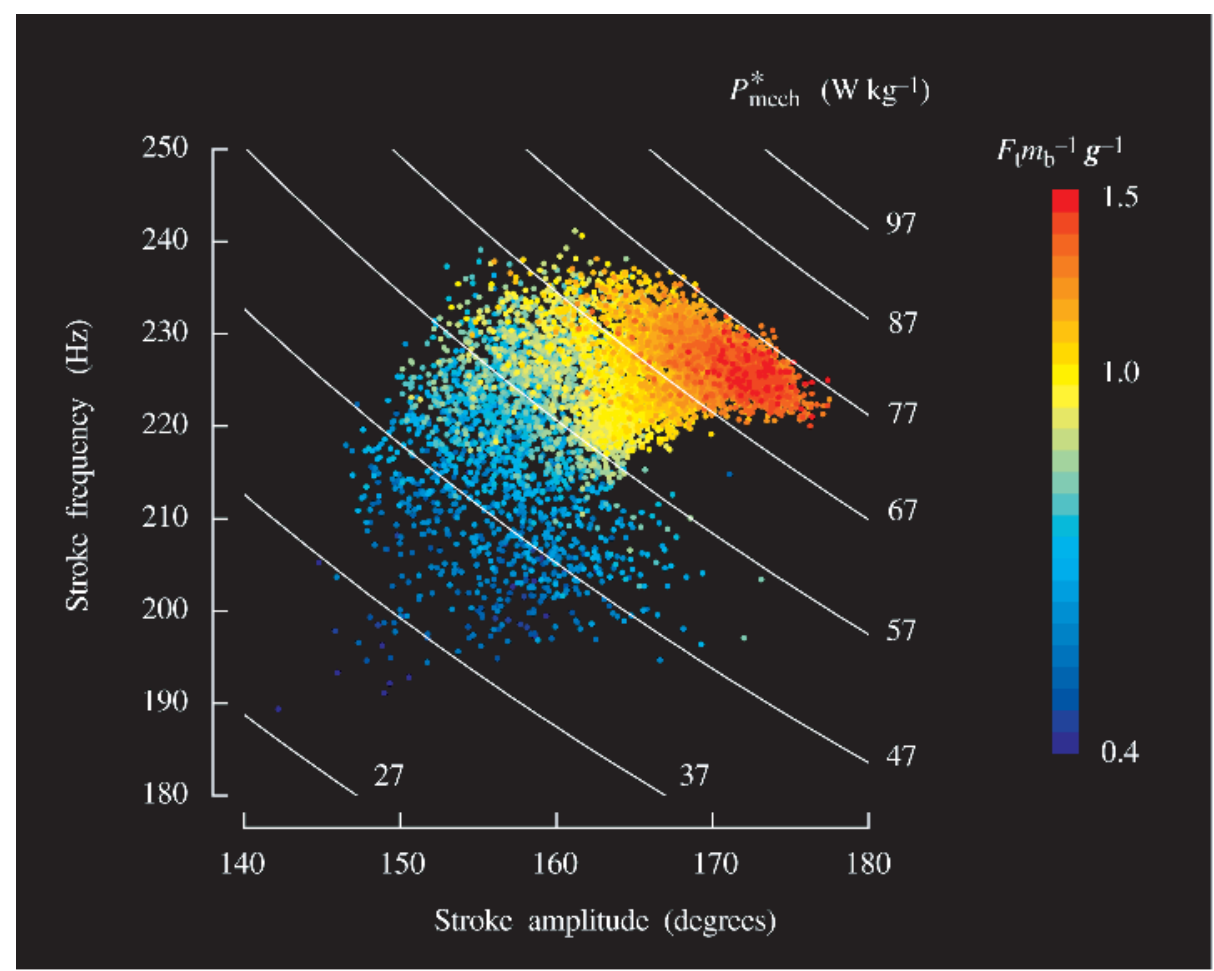


the thorax. Studies in a larger species, $D$. hydei, indicate that elastic storage is at least $10 \%$ and that, under these conditions, only $P_{\text {aero }}^{*}$ contributes to $P_{\text {mech }}^{*}$ (Dickinson and Lighton, 1995). An identical analysis of the current data set led to similar conclusions, and the total mechanical cost of flight in $D$. melanogaster can be safely approximated by $P_{\text {aero. }}^{*}$. However, because $P_{\text {acc }}^{*}$ is close in magnitude to $P_{\text {aero }}^{*}$ throughout the entire range of flight forces, the values of $P_{\text {mech }}^{*}$ calculated under the assumption of $0 \%$ or $100 \%$ elastic storage differ on average by only $6 \%$. The mechanical power rises from a value of $60 \pm 6 \mathrm{~W} \mathrm{~kg}^{-1}$ when flight force is equal to body weight to a maximum value of $77 \pm 12 \mathrm{~W} \mathrm{~kg}^{-1}$.

The maximum mechanical power sustained by the flight muscles represents a constraint on flight kinematics and, thus, on the forces that an animal can produce. The behavioral limits imposed by the maximum value of $P_{\text {mech }}^{*}$ are illustrated in Fig. 7, in which we have superimposed mechanical power isolines over the measured flight force data points in the frequency-amplitude plane. We calculated the isolines for $P_{\text {mech }}^{*}$ using equations 1 and 2 and the morphological parameters for the individual fly. Flight force, required for the calculation of $P_{\text {ind, }}^{*}$, was estimated from wing velocity and a mean lift coefficient of 1.34 , which was determined from the ratio of total flight force to wing velocity when the animal produced enough force to support its body weight. The upper right-hand border of points in Fig. 7 runs along the isoline that represents the maximum power delivered by the flight musculature. The various combinations of amplitude and frequency that a fly can use to modify flight forces must reside to the left of this isoline. In addition, the extreme on the righthand side of this kinematic distribution is constrained by the morphological limit of stroke amplitude.

The maximum total cost was $727 \pm 119 \mathrm{~W} \mathrm{~kg}^{-1}$ compared with a mean resting metabolic rate of $85 \mathrm{~W} \mathrm{~kg}^{-1}$ (both calculated with respect to muscle mass). This results in a metabolic scope of 8.5, which is at the low end of values for other insects (Kammer and Heinrich, 1978). As shown in Fig. 6B, both $P_{\mathrm{CO}_{2}}^{*}$ and $P_{\text {mech }}^{*}$ are linearly correlated with flight force. The efficiency of the flight musculature is determined by the ratio of these two terms (Fig. 6C). In 26 out of the 27 flies, we measured a significant increase $(P<0.001)$ in muscle efficiency over the full range in flight force. Although most of this range includes forces well below that required to support body weight, there was a small but significant difference between the muscle efficiency when force was equal to body weight $(9.1 \pm 1.2 \%)$ and that during maximum force production $(10.7 \pm 1.2 \%)$.

\section{Discussion}

These flight arena experiments have provided several new insights into how the cost of flight changes with an increase in the production of aerodynamic forces. Like all flying animals, flies modulate the production of flight forces by altering their wing stroke kinematics. Although wing motion can change in subtle ways during flight control behavior, stroke amplitude and stroke frequency are probably the most important determinants of flight force in D. melanogaster (Zanker, $1990 b$ ). Our results show that the changes in stroke amplitude and frequency result in a linear increase in wing velocity. This increase has strong effects on the power requirements for flight, since profile power and inertial power are proportional, respectively, to the cube and square of wing velocity. As in previous studies, inertial power is greater than both induced and profile power (Dudley and Ellington, 1990; Ellington, 1984c; Wakeling and Ellington, 1997). However, because the ratio of inertial to aerodynamic power is close to unity, even moderate levels of elastic storage $(<20 \%)$ would be sufficient to eliminate inertial costs (Dickinson and Lighton, 1995). Thus, mechanical power will be determined by the aerodynamic requirements alone. In previous analyses, it has often been assumed that induced power represents the greatest percentage of the total aerodynamic costs (Ellington, 1991; Marden, 1987). For a flying insect within the size range of $D$. melanogaster, however, profile power is roughly twice induced power and represents the major component of the total flight cost.

\section{Mechanical power}

The results suggest that $D$. melanogaster indirect flight muscles are capable of a maximum mechanical power output of just under $80 \mathrm{~W} \mathrm{~kg}^{-1}$ at a temperature of $24^{\circ} \mathrm{C}$. Even if we take the free flight experiments at face value and assume that untethered flies can generate $20 \%$ more force, we would still predict a maximum output of no greater than $100 \mathrm{~W} \mathrm{~kg}^{-1}$. This is only $63 \%$ of the maximum mechanical output in three euglossine bees (Euglossa dissimula, Euglossa imperialis and Eulaema meriana) estimated from an analysis using helium-oxygen mixtures (Dudley, 1995). However, Marden's $(1987,1994)$ study of maximum take-off weights indicates that peak mechanical power is strongly size-dependent. Marden's original data were based solely on induced power and were subsequently increased (Ellington, 1991; Marden, 1994) by a size-invariant $25 \%$ to account for the contribution of profile power. On the basis of the uncorrected scaling data, an animal the size of D. melanogaster should possess a peak mechanical power of approximately $34 \mathrm{~W} \mathrm{~kg}^{-1}$, which agrees closely with our measurement of peak induced power $\left(32 \mathrm{~W} \mathrm{~kg}^{-1}\right)$. However, in the current experiments, the profile power is roughly twice the induced power, which suggests that the $25 \%$ correction of Marden's data is much too low to account for the profile power requirements of animals in the size range of $D$. melanogaster.

\section{Muscle efficiency}

Several in vivo estimates of mechanical efficiency have been made for a variety of insect flight muscles. These include $11 \%$ for Drosophila hydei (Dickinson and Lighton, 1995), 4-16\% for various euglossine bees (Casey and Ellington, 1989), $10 \%$ for Manduca sexta (Stevenson and Josephson, 1990), 12.6\% for Sympetrum sanguineum and $8.7 \%$ for Calopteryx splendens (Wakeling and Ellington, 1997). The only direct in 
vitro measurement for insect flight muscle is $6 \%$ in Schistocerca americana (Josephson and Stevenson, 1991). All these values cluster around $10 \%$ and there appears to be little difference between synchronous and asynchronous muscles, despite the supposedly high cost of $\mathrm{Ca}^{2+}$ cycling (Homsher and Kean, 1978). The value of $9 \%$ reported here for hovering flight in D. melanogaster is consistent with these previous measurements. However, if we were to assume, a priori, that $9-10 \%$ is a reasonable value for the mechanical efficiency of Drosophila flight muscles, then the respirometric measurements of $P_{\mathrm{CO}_{2}}^{*}$ lend credence to our kinematic

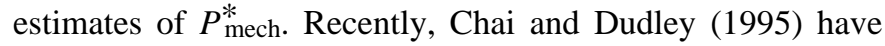
estimated the efficiency of hummingbird muscle to be $10 \%$, which might suggest that the general constraint of low efficiency found in insect flight muscles extends to vertebrates as well.

The results shown in Fig. 6C indicate that muscle efficiency rises with increasing flight force. Because of the many possible errors in the calculation of mechanical power, it is important to rule out the possibility that this increase is simply an artifact of an inaccurate power estimate. A constant value of muscle efficiency would imply that our estimates greatly overestimate

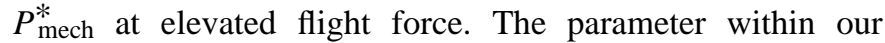
calculations that is most prone to error is the mean profile drag coefficient, which is based on a steady-state approximation of profile drag based on Reynolds number. However, recent experiments with the flight arena (F.-O. Lehmann and M. H. Dickinson, in preparation) indicate that the mean force coefficient (a vector sum of the lift and drag coefficients) is greater than can be explained by steady-state mechanisms and rises with increasing force production. For this reason, it is more likely that we underestimate $P_{\text {mech }}^{*}$ at elevated flight forces and that the rise in muscle efficiency may, in fact, be steeper than our present calculations would indicate. We therefore conclude that the observed rise in efficiency with increasing flight force represents a real physiological alteration within the flight musculature. This increase could result from the changes in magnitude or rate of strain in the indirect power muscles that accompany the elevation in wing velocity. In addition, since flies are known to increase the spike rate of the motor neurons innervating the power muscles in response to a visual lift stimulus (Heide, 1983; Heide et al. 1985; Smyth and Yurkiewicz, 1966), the activation of crossbridges may increase at elevated flight forces. Since the asynchronous power muscles have a prominent voltage-gated $\mathrm{Ca}^{2+}$ conductance (Salkoff and Wyman, 1983), the increased motor drive is likely to increase cytosolic $\left[\mathrm{Ca}^{2+}\right]$ even in the absence of extensive sarcoplasmic stores. Muscle efficiency might increase as the number of active crossbridges rises.

Although the change in muscle efficiency between hovering and peak force production is rather small (Table 2), the larger change in efficiency over the full range of measured forces might have important implications for the energetics of forward flight. According to several well-established models of flight energetics, total mechanical power is expected first to decline and then to increase with increasing flight speed, creating a U-shaped cost curve (Pennycuick, 1968; Rayner, 1979; Tucker, 1973). However, several investigations of birds, bats and insects have found that total metabolic rate is nearly independent of forward speed and follows a more J-shaped curve (Ellington, 1991). One explanation for this discrepancy is that muscle efficiency is strain- and activation-dependent in a way that might lead to higher efficiency, and thus lower measured metabolic cost, under near-hovering conditions. The current results support this hypothesis, since the kinematics that produce flight forces below body weight (and low muscle efficiency) may be closer to those that occur during forward flight, when force production by the wings is augmented by the translational velocity of the body. Muscle efficiency appears to be greatest at the elevated wing velocity and the corresponding muscle strain rate characteristic of hovering flight.

This project was generously funded by a Packard Foundation Fellowship and NSF grant IBN-9208765 (to M.H.D.) and a DFG Post-Doctoral Fellowship (to F.-O.L.). The authors wish to acknowledge David Smith, Gerard Jendraszkiewicz and Brian Goers of the James Franck Institute at the University of Chicago for their work in the design and construction of the flight arena and Jeff Hamlin for his help with morphometric measurements.

\section{References}

CAsey, T. M. (1981). A comparison of mechanical and energetic estimates of flight cost for hovering sphinx moths. J. exp. Biol. 91, 117-129.

Casey, T. M. and Ellington, C. P. (1989). Energetics of insect flight. In Energy Transformations in Cells and Organisms (ed. W. Wieser and E. Gnaiger), pp. 200-210. Stüttgart: Thieme Verlag.

ChaI, P. AND Dudley, R. (1995). Limits to vertebrate locomotor energetics suggested by hummingbirds hovering in heliox. Nature 377, 722-725.

DAVID, C. T. (1978). The relationship between body angle and flight speed in free flying Drosophila. Physiol. Ent. 3, 191-195.

Dickinson, M. H. AND GöTZ, K. G. (1993). Unsteady aerodynamic performance of model wings at low Reynolds numbers. J. exp. Biol. 174, 45-64.

Dickinson, M. H. AND GöTz, K. G. (1996). The wake dynamics and flight forces of the fruit fly Drosophila melanogaster. J. exp. Biol. 199, 2085-2104.

Dickinson, M. H., Lehmann, F.-O. And Götz, K. G. (1993). The active control of wing rotation by Drosophila. J. exp. Biol. 182, 173-189.

Dickinson, M. H. And Lighton, J. R. B. (1995). Muscle efficiency and elastic storage in the flight motor of Drosophila. Science $\mathbf{1 2 8}$, 87-89.

DudLEY, R. (1990). Biomechanics of flight in neotropical butterflies: morphometrics and kinematics. J. exp. Biol. 150, 37-53.

DUDLEY, R. (1995). Extraordinary flight performance of orchid bees (Apidae: Euglossini) hovering in heliox $\left(80 \% \mathrm{He} / 20 \% \mathrm{O}_{2}\right)$. J. exp. Biol. 198, 1065-1070.

Dudley, R. And Ellington, C. (1990). Mechanics of forward flight in bumblebees. II. Quasi-steady lift and power requirements. J. exp. Biol. 148, 53-88. 
Ellington, C. P. (1984a). The aerodynamics of insect flight. II. Morphological parameters. Phil. Trans. R. Soc. Lond. B 305, $17-40$.

Ellington, C. P. (1984b). The aerodynamics of insect flight. V. A vortex theory. Phil. Trans. R. Soc. Lond. B 305, 115-144.

Ellington, C. P. (1984c). The aerodynamics of insect flight. VI. Lift and power requirements. Phil. Trans. R. Soc. Lond. B 305, $145-181$.

Ellington, C. P. (1991). Limitations on animal flight performance. J. exp. Biol. 160, 71-91.

Ellington, C. P., Machin, K. E. And Casey, T. M. (1990). Oxygen consumption of bumblebees in forward flight: the $U$-shaped curve is flat. Nature 347, 472-473.

Götz, K. G. (1987). Course-control, metabolism and wing interference during ultralong tethered flight in Drosophila melanogaster. J. exp. Biol. 128, 35-46.

GöTZ, K. G. AND WANDEL, U. (1984). Optomotor control of the force of flight in Drosophila and Musca. II. Covariance of lift and thrust in still air. Biol. Cybernetics 51, 135-139.

HeIDE, G. (1983). Neural mechanisms of flight control in Diptera. In BIONA-Report 2 (ed. W. Nachtigall), pp. 35-52. Stuttgart: Fischer.

Heide, G., SPÜLER, M., Götz, K. G. AND KAMPER, K. (1985). Neural control of asynchronous flight muscles in flies during induced flight manoeuvers. In Insect Locomotion (ed. G. Wendler), pp. 215-222. Berlin: Paul Parey.

HeisenberG, M. AND Wolf, R. (1984). Vision in Drosophila. Berlin: Springer-Verlag.

Homsher, E. And Kean, C. J. (1978). Skeletal muscle energetics and metabolism. A. Rev. Physiol. 40, 93-131.

JoSEPHSON, R. K. (1993). Contraction dynamics and power output of skeletal muscle. A. Rev. Physiol. 55, 527-546.

Josephson, R. K. And Stevenson, R. D. (1991). The efficiency of a flight muscle from the locust, Schistocerca americana. J. Physiol., Lond. 442, 413-429.

Kammer, A. E. AND HeInRICh, B. (1978). Insect flight metabolism. Adv. Insect Physiol. 13, 133-228.

LEHMANN, F.-O. (1994). Aerodynamische, kinematische und electrophysiologische Aspekte der Flugkrafterzeugung und
Flugkraftsteuerung bei der Taufliege Drosophila melanogaster. Thesis, Eberhad-Karls-Universität Tübingen.

Lighton, J. R. B. (1991). Insect metabolism. In Concise Encyclopedia of Biological and Biomedical Measurement Systems (ed. P. A. Payne), pp. 201-208. New York: Pergamon.

MARDEN, J. (1987). Maximum lift production during take-off in flying animals. J. exp. Biol. 130, 235-258.

MARDEN, J. H. (1994). From damselflies to pterosaurs: how burst and sustainable flight performance scale with size. Am. J. Physiol. 266, 1077-1084.

PenNyCuick, C. J. (1968). Power requirements for horizontal flight in the pigeon Columba livia. J. exp. Biol. 49, 527-555.

RAYNER, J. M. V. (1979). A new approach to animal flight mechanics. J. exp. Biol. 80, 17-54.

RAYNER, J. M. V. (1991). On the aerodynamics of animal flight in ground effect. Phil. Trans. R. Soc. Lond. B 334, 119-128.

RAYNER, J. M. V. (1994). Aerodynamic corrections for the flight of bats and birds in wind tunnels. J. Zool., Lond. 234, 537-563.

SALKOFF, L. AND WyMAN, R. (1983). Ionic currents in Drosophila. J. Physiol., Lond. 337, 687-709.

Smyth, T. AND Yurkiewicz, W. J. (1966). Visual reflex control of indirect flight muscles in the sheep blowfly. Comp. Biochem. Physiol. 17, 1175-1180.

SteVEnson, R. D. AND Josephson, R. K. (1990). Effects of operating frequency and temperature on mechanical power output from moth flight muscle. J. exp. Biol. 149, 61-78.

TUCKER, V. A. (1973). Bird metabolism during flight: evaluation of theory. J. exp. Biol. 58, 689-709.

Vogel, S. (1966). Flight in Drosophila. I. Flight performance of tethered flies. J. exp. Biol. 44, 567-578.

Vogel, S. (1967). Flight in Drosophila. III. Aerodynamic characteristics of fly wings and wing models. J. exp. Biol. 46, 431-443.

Wakeling, J. M. And Ellington, C. P. (1997). Dragonfly flight. III. Lift and power requirements. J. exp. Biol. 200, 557-582.

ZANKER, J. M. (1990a). The wing beat of Drosophila melanogaster. I. Kinematics. Phil. Trans. R. Soc. Lond. B 327, 1-18.

ZANKER, J. M. (1990b). The wing beat of Drosophila melanogaster. III. Control. Phil. Trans. R. Soc. Lond. B 327, 45-64. 